\title{
Potential Role of Biomarkers in the Management of Syncope
}

\author{
Birsen Arici ${ }^{*}$ Mirjam Maeder*, Philipp Schuetz, Beat Mueller", Werner C. Albrich ${ }^{\#}$
}

Medical University Department of the University of Basel, Kantonsspital Aarau, Switzerland.

Email: BArici@uhbs.ch

Received October $6^{\text {th }}, 2012$; revised November $15^{\text {th }}, 2012$; accepted November $24^{\text {th }}, 2012$

\begin{abstract}
Study Objective: Syncope is one of the most common presentation of patients seen in emergency departments (ED). Risk assessment of syncope is challenging. The San Francisco Syncope Rule is the most widely used risk assessment, but has moderate accuracy. The aim of our study was to investigate blood biomarkers as prognostic factors for adverse outcome. Methods: In this observational study we included consecutive adults presenting with syncope to our ED. Management decisions were left to the discretion of the treating physicians. Patients were monitored for adverse events until discharge and underwent a phone interview 30 days after enrolment. Adverse outcome was defined as recurrent syncope, rehospitalization and death within 30 days. Results: We included 132 adult patients of whom 19 (14\%) had an adverse event (recurrent syncope $=3$, rehospitalisation $=12$, death $=4)$. No difference in the San Francisco Syncope Rule was found in patients with and without adverse events (SFSR $\geq 1: 37 \%$ vs $39 \% ; p=0.877)$. Median levels of ProADM (1.23 vs $0.81 \mathrm{nmol} / 1 ; \mathrm{p}=0.006)$ and NT-proBNP (454 vs $134 \mathrm{ng} / 1 ; \mathrm{p}=0.035)$ were higher and median levels for cholesterol ( 3.68 vs $4.57 \mathrm{mmol} / \mathrm{l} ; \mathrm{p}=0.008)$ and prealbumin $(0.19 \mathrm{vs} 0.26 \mathrm{~g} / \mathrm{l} ; \mathrm{p}=0.005)$ were lower in patients with adverse events. Prealbumin (AUC 0.72) and ProADM (AUC 0.70) had the highest prognostic accuracy. Conclusion: Biomarkers predicted poor outcome and might be helpful in the context of a clinical algorithm for an improved triage of syncope patients in the ED.
\end{abstract}

Keywords: Blood Biomarkers; Syncope; San Francisco Syncope Rule

\section{Introduction}

Syncope is a common symptom among patients presenting to emergency departments (EDs) [1]. The most frequently identified cause of syncope is neurally-mediated (neurocardiogenic syncope), which is not associated with an increased risk of cardiovascular morbidity and mortality. On the other hand risk of death is doubled among patients with cardiac syncope as compared to those without syncope [2]. Although short-term serious adverse events are rare, more than half of patients are hospitalized for evaluation according to European Society of Cardiology (ESC) guidelines [3] and $25 \%$ of the patients are hospitalized without clear indication [4]. Despite extensive testing in hospitalized patients the yield of diagnostic tests is low especially in older patients [5] and the total annual costs of syncope-related admissions exceed \$2 billion in the United States [6].

Several predictors of short-term serious events after syncope and clinical decision rules in syncope-evaluation have been described [7-9]. The San Francisco Syncope

\footnotetext{
*Equally contributing first authors.

\#Equally contributing senior authors.
}

Rule (SFSR) is the most thoroughly investigated prediction rule for the assessment of syncope [10], albeit with inconsistent results in validation studies according to a recent systematic review [11].

Thus, there is a lack of a reliable, widely accepted and universal risk prediction tool for serious outcomes in patients with syncope. Clinical evaluation and treatment of these patients vary widely with the healthcare setting, experience and knowledge of the treating physician and is not always consistent with guidelines. In fact, uncertainty of health-care professionals about risks and emotional concerns of patients and relatives about seriousness or other biopsychosocial causes frequently lead to unnecessary admissions. Beyond history and clinical information objective parameters are desirable, which should be simple, readily available and cost-effective, to improve management and allow discharge of low-risk syncope patients from the ED.

The aim of this study was to evaluate the prognostic value of biomarkers in patients presenting with syncope to the ED. For this purpose we conducted a cohort study of patients with syncope presenting to the ED and investigated the association of different initial blood bio- 
markers with the outcome within 30 days of follow up.

\section{Methods}

\subsection{Study Design}

This was a single-center prospective observational study to evaluate the prognostic value of blood biomarkers for syncope at a Medical University Clinic in Aarau in central Switzerland. Consecutive adult patients presenting to the ED with syncope between November 2010 and August 2011 were included. Syncope was defined as transient but complete loss of consciousness of rapid onset, short duration and spontaneous recovery. Exclusion criteria were as follows: syncope due to pulmonary embolism, acute myocardial infarction, myocarditis, endocarditis, acute cardiac trauma, acute valvular heart disease, craniocerebral injury, acute stroke, epileptic seizure, intoxication, hypoglycemia, near syncope, acute cardiac failure and history of heart surgery within one month.

A standard questionnaire and a standardized physical examination were completed by medicine residents in the ED. Biomarkers which were considered to be relevant in syncope (troponin I, NT-proBNP, Proadrenomedullin (ProADM) were measured in all patients using comercial assays. In addition we measured cortisol as stress hormone and cholesterin, TSH, folic acid, vitamin B12, vitamin D, prealbumin, IGF-1, hGH, ferritin and transferrin as endocrine or nutritional markers for general health and nutrition status.

ProADM were blinded to the treating physicians. Management decisions were left to the discretion of the treating physician without influence by the study team.

Patients were monitored from admission to hospital discharge. Diagnostic and therapeutic procedures were recorded. All patients, or their relatives if patients were unable to provide information, underwent a follow-up phone interview 30 days after enrolment. If an adverse event was recorded or information was not clear, confirmation was sought from the general practitioner.

This survey was part of a large quality improvement project at the Kantonsspital Aarau. The local Institutional Review Board (Kantonale Ethikkommission Aargau) classified this study as an observational quality surveillance and waived the need for patient informed consent (EKAG: 2009/074).

\subsection{Medical Risk}

The SFSR is a risk assessment tool for adverse events that consists of the following items [10]: history of congestive heart failure, hematocrit $<30 \%$, abnormal electrocardiogram (ECG), shortness of breath and triage systolic blood pressure $<90 \mathrm{mmHg}$. A patient with any of these was considered to be at high risk for an adverse event, and low otherwise. Serious outcome in the validation study was defined as death, myocardial infarction, arrhythmia, pulmonary embolism, stroke, subarachnoid hemorrhage, significant hemorrhage, or any condition causing a return ED visit and hospitalization for a related event.

In our study SFSR was calculated for every patient by the study team, but the results were not available for the treating physicians. For simplification we concentrated the adverse events to recurrent syncope, rehospitalization and death.

\subsection{Methods of ProADM Measurement}

ProADM was batch-analysed from EDTA serum from the routinely collected blood specimen at admission using a sandwich immunoassay with an analytical detection limit of $0.08 \mathrm{mmol} / \mathrm{l}$.

\subsection{Causes of Syncope}

The etiology of the syncopal episodes was determined on the basis of all available test results as determined by the treating physician team. Patients were classified into the following categories: neurally-mediated (vasovagal, neurocardiogenic), orthostatic, cardiovascular. If the cause was unclear or psychogenic the syncope was classified as unexplained.

A ECG was considered pathological if it showed any of the following abnormalities: bifascicular block, ORSduration $\geq 0.12$ seconds, second or third degree atriventricular (AV) block, sinus bradycardia $<50 /$ min, sinuatrial block or sinus pause $>3$ seconds, non-sustained ventricular tachycardia (VT), pre-excited QRS complexes, long or short QT intervals, early repolarisation, right bundle branch block (RBBB) pattern with ST-elevation in leads V1-V3 (Brugada syndrome), negative $\mathrm{T}$ waves in right precordial leads, epsilon waves and ventricular late potentials suggestive of arrhythmogenic right ventricular cardiomyopathy (ARVC) and Q waves suggesting myocardial infarction.

\subsection{Endpoints}

The primary endpoint was the occurrence of any adverse event within 30 days of follow up. Adverse outcome were evaluated during hospitalization and with telephone interview 30 days after enrolment. They included recurrent syncope as reported by the patient, re-hospitalization for any cause and death from any cause.

\subsection{Statistical Analyses}

Discrete variables were expressed as counts (percentage) and continuous variables as means (standard deviation) or medians (interquartile range (IQR)) as appropriate. For two-group comparison the non-parameteric MannWhitney-U test was used. To evaluate the association of markers with the primary endpoint, a logistic regression 
analysis was done. The discriminatory value was assessed in receiver operating curve analysis (ROC). All statistical tests were done using SPSS (version 10.0 and 20 for windows, IBM, USA) and SAS (SAS Institute, Cary, NC, USA). A $p$ value $\leq 0.05$ was considered statistically significant.

\section{Results}

\subsection{Baseline Characteristics}

We included 132 patients presenting with syncope to the ED: Mean age was 65 years, 54\% were male (Table 1). Patients with adverse events tended to be older and to have a higher Charlson comorbidity index, but the differences were not significant. Medical history did not differ in both groups except for arrhythmia, which was more common in patients with adverse events $(37 \%$ vs $15 \%, \mathrm{p}=$ 0.039 ). The proportion of patients with pathological ECG was similar $(16 \%$ vs $24 \%, p=0.465)$ in patients with and those without adverse events.

\subsection{Primary Endpoint}

A total of 19 patients (14\%) had an adverse event within 30 days. Three patients $(2 \%)$ had another episode of syncope without need for hospitalization. Twelve patients $(9 \%)$ had a re-hospitalisation and four patients (3\%) died. Causes of death were cardiac $(\mathrm{n}=1)$, sepsis $(\mathrm{n}=1)$ and malignancy $(\mathrm{n}=2)$.

$15 \%$ of the patients, which were classified in the SFSR as low risk population and $14 \%$ of the patient, which were classified in the SFSR as high risk population had an adverse events within 30 days (Table 2). Overall, the accuracy of the SFSR was low (AUC 0.52). At the recommended cut off of SFSR $\geq 1$, the sensitivity to predict adverse event was $37 \%$ with specificity of $61 \%$.

\subsection{Specific Testing}

Specific diagnostic tests were obtained for evaluation of syncope: telemetry was performed in $51 \%$ of the patients and relevant pathological findings were revealed in $41 \%$

Table 1. Baseline characteristics.

\begin{tabular}{|c|c|c|c|c|}
\hline \multirow{2}{*}{ Characteristics } & All patients & No adverse events & Adverse events & \multirow{2}{*}{$\mathbf{p}$} \\
\hline & $(n=132)$ & $(n=113)$ & $(n=19)$ & \\
\hline Sex & & & & 0.913 \\
\hline Male, no. $(\%)$ & $71(54 \%)$ & $61(86 \%)$ & $10(14 \%)$ & \\
\hline Female, no. $(\%)$ & $61(46 \%)$ & $52(85 \%)$ & $9(15 \%)$ & \\
\hline Charlson comorbidity index, median (IQR) & $4(5.00)$ & $3(5.00)$ & $5(6.00)$ & 0.169 \\
\hline \multicolumn{5}{|l|}{ Medical history, no. (\%) } \\
\hline Syncope & $61(46 \%)$ & $53(47 \%)$ & $8(42 \%)$ & 0.710 \\
\hline Arterial hypertension & $72(54 \%)$ & $60(53 \%)$ & $12(63 \%)$ & 0.431 \\
\hline Myocardial infarction & $18(14 \%)$ & $14(12 \%)$ & $4(21 \%)$ & 0.334 \\
\hline Cerebrovascular disease & $14(11 \%)$ & $12(11 \%)$ & $2(11 \%)$ & 0.958 \\
\hline Peripheral artery disease & $10(8 \%)$ & $7(6 \%)$ & $3(16 \%)$ & 0.194 \\
\hline Arrhythmia & $24(18 \%)$ & $17(15 \%)$ & $7(37 \%)$ & 0.039 \\
\hline Defibrillator/pacemaker & $8(6 \%)$ & $5(4 \%)$ & $3(16 \%)$ & 0.104 \\
\hline Congestive heart failure & $15(11 \%)$ & $13(11 \%)$ & $2(11 \%)$ & 0.957 \\
\hline Valvular heart disease & $14(11 \%)$ & $14(12 \%)$ & $0(0 \%)$ & 0.438 \\
\hline Diabetes & $19(14 \%)$ & $16(14 \%)$ & $3(16 \%)$ & 0.821 \\
\hline Psychiatric disorders & $13(10 \%)$ & $9(8 \%)$ & $4(21 \%)$ & 0.116 \\
\hline Pathologic ECG, no. (\%) & $30(23 \%)$ & $27(24 \%)$ & $3(16 \%)$ & 0.465 \\
\hline SFSR $\geq 1$, no. $(\%)$ & $51(39 \%)$ & $44(39 \%)$ & $7(37 \%)$ & 0.877 \\
\hline
\end{tabular}


Table 2. Adverse events within 30 days after enrollment.

\begin{tabular}{cccc}
\hline & $\begin{array}{c}\text { All } \\
\text { patients }\end{array}$ & $\begin{array}{c}\text { SFSR }=\mathbf{0} \\
\text { (low risk) }\end{array}$ & $\begin{array}{c}\text { SFSR } \geq \mathbf{1} \\
\text { (high risk) }\end{array}$ \\
\cline { 2 - 4 }$(\mathbf{n}=\mathbf{1 3 2})$ & $(\mathbf{n}=\mathbf{8 1})$ & $(\mathbf{n}=\mathbf{5 1})$ \\
\hline All adverse events & $19(14 \%)$ & $12(15 \%)$ & $7(14 \%)$ \\
Resyncope & $3(2 \%)$ & $3(4 \%)$ & $0(0 \%)$ \\
$\begin{array}{c}\text { Readmission } \\
\text { Death from any } \\
\text { cause }\end{array}$ & $12(9 \%)$ & $8(10 \%)$ & $4(8 \%)$ \\
\hline
\end{tabular}

of these evaluated patients, echocardiography in $32 \%$ with pathological findings in $20 \%$, Schellong test (fall of systolic pressure of $20 \mathrm{mmHg}$ while standing indicates orthostasis) in $80 \%$ with pathological findings in $31 \%$, head CT in $17 \%$ with pathological findings in $14 \%$, carotid ultrasound in 13\% with pathological findings in 20\%, cardiac stress test and EEG both in $10 \%$ with no pathological findings.

\subsection{Causes of Syncope}

The most common etiology of syncope was orthostatic (43.2\%), followed by neurally-mediated syncope $(28.8 \%)$ and cardiovascular syncope (16.7\%). Only $11.4 \%$ of the cases remained unexplained.

\subsection{Biomarkers}

Median levels of ProADM (1.23 vs $0.81 \mathrm{nmol} / \mathrm{l} ; \mathrm{p}=0.006$ ) and NT-proBNP (454 vs $134 \mathrm{ng} / \mathrm{l} ; \mathrm{p}=0.035$ ) were higher and median levels for cholesterol (3.68 vs $4.57 \mathrm{mmol} / \mathrm{l} ; \mathrm{p}=$ $0.008)$ and prealbumin ( 0.19 vs $0.26 \mathrm{~g} / \mathrm{l} ; \mathrm{p}=0.005)$ were lower in patients with compared to those without adverse events (Table 3).

Prealbumin (AUC 0.28 resp. 0.72) and ProADM (AUC $0.70)$ had the highest prognostic accuracy for prediction of adverse events.

To test the prognostic accuracy of different markers together, we calculated multivariate logistic regression analysis. A combined score (Figure 1) including SFSR, prealbumin and ProADM yielded a high discriminatory ability with an AUC of 0.75 (95\% CI 0.62 - 0.88).

We divided the values of prealbumin and ProADM in tertiles (Figures 2 and 3). ProADM equal to or greater than $0.7 \mathrm{nmol} / 1$ (second and third tertile) had a sensitivity of $83 \%$ for adverse events in the low risk population (according to SFSR) and a sensitivity of $100 \%$ in the high risk population (according to SFSR). Prealbumin greater than $0.29 \mathrm{~g} / \mathrm{l}$ (third tertile) had a specificity of $89 \%$ in the low risk and $86 \%$ in the high risk population.

Median level of ProADM in the group of patients with neurally-mediated syncope $(0.67 \mathrm{nmol} / \mathrm{l})$ was significantly lower compared to the other causes of syncope (1.02

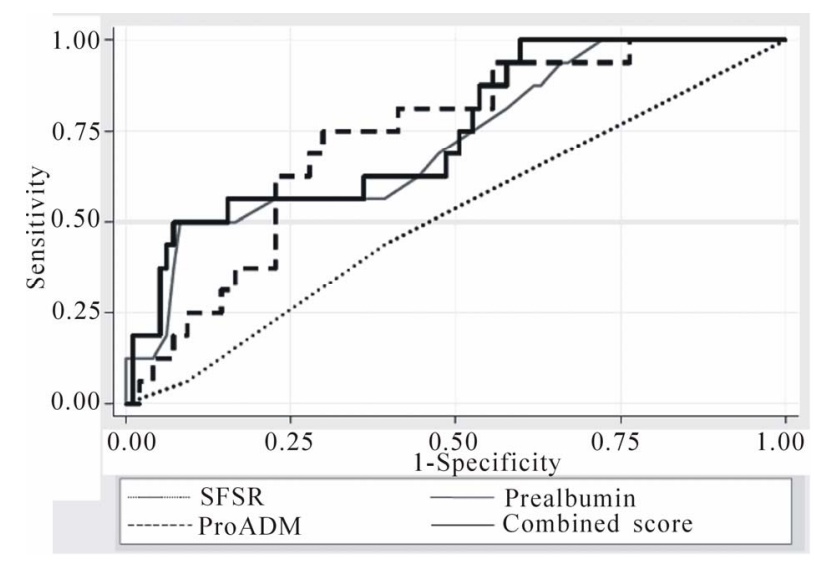

Figure 1. ROC curves for SFSR, ProADM, prealbumin and a combined score.

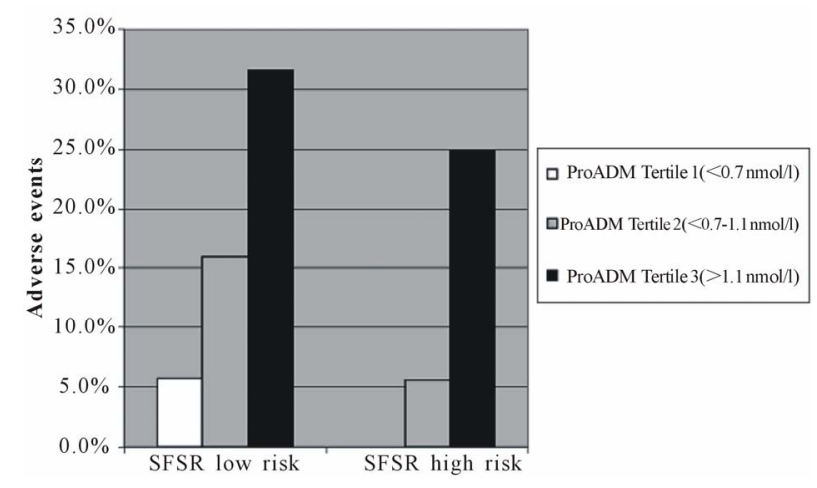

Figure 2. Distribution of adverse events regarding to SFSR risk and ProADM.

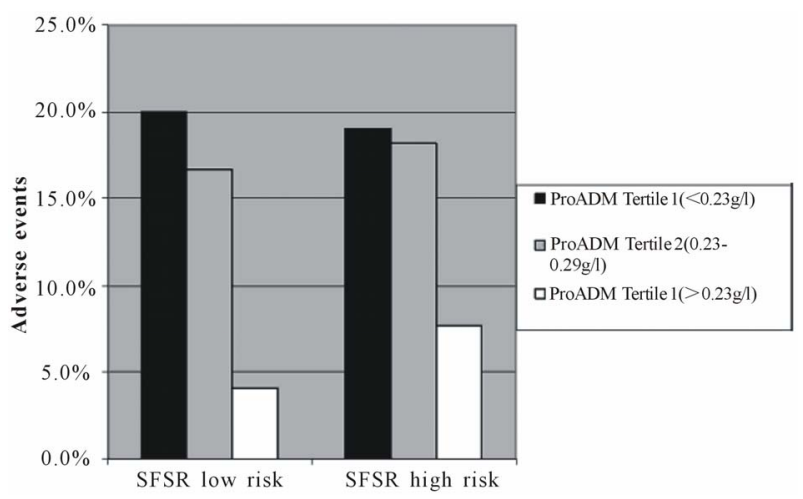

Figure 3. Distribution of adverse events regarding to SFSR risk and Prealbumin.

$\mathrm{nmol} / \mathrm{l} ; \mathrm{p}=0.0011)$ (Figure 4). In contrast, median levels of prealbumin did not differ in the various causes of syncope $(0.26,0.23,0.26$ vs $0.24 ; p=0.06)$.

\section{Discussion}

In this observational study of patients presenting with syncope to the ED of a Swiss medical university hospital we found prealbumin and ProADM to be predictors of 
adverse outcome during a 30-days follow-up. While the SFSR alone did not have a high prognostic ability, the addition of biomarkers improved its performance.

Many efforts have been made to find criteria that could safely differentiate benign from harmful outcome in syncope patient. The SFSR is the most thoroughly investigated prediction rule for the assessment of syncope and showed in the first validation study a sensitivity of $98 \%$ and specificity of $56 \%$ to predict adverse events within 30 days $[10]$.

A recently published systematic review showed inconsistent results in validation studies of the SFSR [11]. Sun et al. found a sensitivity of $89 \%$ and specificity of $42 \%$ and Birnbaum et al. an even lower sensitivity of $74 \%$ and

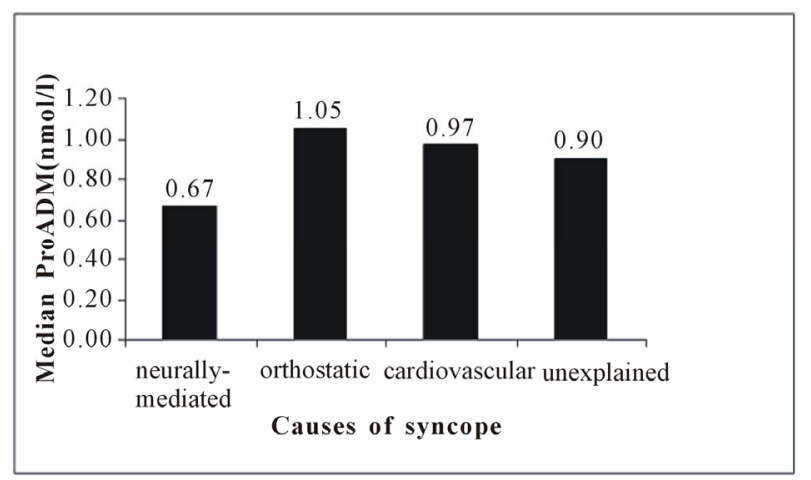

Figure 4. ProADM levels in different causes of syncope. specificity of $57 \%[12,13]$. In our study the ability of the SFSR with a recommended cut off of $\geq 1$ for predicing adverse outcome was insufficient with a sensitivity of $37 \%$ and specificity of $61 \%$.

A recently published prospective cohort study showed that another risk tool (Boston Syncope Criteria) was able to safely reduce admissions [14]. Limitations for clinical routine are the large number of items ( 25 items, 8 categories) and the lower specificity of this new tool.

Despite clinical guidelines the number of unnecessary hospitalisations remains high because of unclear aetiology and for fear of missing life-threatening conditions.

Therefore new decision tools - other than history taking, physical examination and ECG-are needed to improve the management of syncope in the emergency departments. These should make it easier to discharge patients safely and reduce costs.

In the BACH study, a prospective, multicenter study of 1641 patients presenting to the ED with dyspnoea ProADM identified patients with high 90-day mortality. ProADM was superior to predict all-cause mortality in acute shortness of breath compared with B-type natriuretic peptide or troponin [15]. The LAMP II study [16] showed that ProADM was a better prognostic marker for death or heart failure in patients with non-ST-elevation myocardial infarction compared with NT-proBNP or GRACE risk score.

Table 3. Baseline biomarkers.

\begin{tabular}{|c|c|c|c|c|c|c|c|c|c|}
\hline \multirow{3}{*}{$\begin{array}{c}\text { Biomarkers, median (IQR) } \\
\text { NTpro-BNP (ng/l) }\end{array}$} & \multirow{2}{*}{\multicolumn{2}{|c|}{$\begin{array}{c}\text { All patients } \\
(n=132)\end{array}$}} & \multirow{2}{*}{\multicolumn{2}{|c|}{$\begin{array}{c}\text { no adverse events } \\
(n=113)\end{array}$}} & \multirow{2}{*}{\multicolumn{2}{|c|}{$\begin{array}{c}\text { adverse events } \\
(n=19)\end{array}$}} & \multirow{3}{*}{$\begin{array}{l}\mathbf{O R}^{*} \\
2.009\end{array}$} & \multirow{3}{*}{$\begin{array}{r}\text { p-value } \\
0.035\end{array}$} & \multirow{3}{*}{$\begin{array}{l}\text { AUC } \\
0.651\end{array}$} \\
\hline & & & & & & & & & \\
\hline & 159.50 & $(660.25)$ & 134.00 & (490.00) & 454.00 & (1001.00) & & & \\
\hline ProADM (nmol/l) & 0.87 & $(0.65)$ & 0.81 & $(0.61)$ & 1.23 & $(0.64)$ & 14.96 & 0.006 & 0.699 \\
\hline Troponin I (ug/l) & 0.02 & (0) & 0.02 & (0) & 0.02 & (0) & 2.344 & 0.073 & 0.567 \\
\hline Cortisol (nmol/l) & 656.50 & $(542.75)$ & 651.00 & $(582.00)$ & 668.00 & $(441.00)$ & 1.36 & 0.600 & 0.538 \\
\hline Cholesterin (mmol/l) & 4.47 & $(1.74)$ & 4.57 & $(1.57)$ & 3.68 & (2.09) & 0.001 & 0.008 & 0.311 \\
\hline TSH (mU/l) & 1.83 & $(2.09)$ & 1.85 & $(2.40)$ & 1.62 & $(2.02)$ & 0.564 & 0.369 & 0.435 \\
\hline Folic acid (nmol/l) & 17.70 & (13.10) & 17.90 & $(14.00)$ & 15.40 & $(14.20)$ & 0.472 & 0.401 & 0.436 \\
\hline Vitamin B12 (pmol/1) & 325.00 & $(239.00)$ & 326.00 & $(225.00)$ & 321.00 & $(265.00)$ & 0.546 & 0.563 & 0.458 \\
\hline Vitamin D (nmol/1) & 47.90 & $(48.15)$ & 52.75 & $(49.60)$ & 40.00 & $(22.90)$ & 0.1 & 0.049 & 0.358 \\
\hline Prealbumin (g/l) & 0.26 & $(0.09)$ & 0.26 & $(0.09)$ & 0.19 & $(0.09)$ & 0.002 & 0.005 & 0.279 \\
\hline IGF-1 (nmol/1) & 13.85 & $(10.27)$ & 14.00 & $(10.10)$ & 11.40 & (9.13) & 0.181 & 0.099 & 0.381 \\
\hline Ferritin (ug/l) & 129.00 & $(219.50)$ & 122.00 & $(200.00)$ & 170.00 & $(314.00)$ & 1.526 & 0.208 & 0.591 \\
\hline Transferrin (umol/l) & 28.60 & $(7.10)$ & 28.50 & $(6.40)$ & 29.30 & $(10.70)$ & 0.04 & 0.772 & 0.479 \\
\hline hGH (ug/l) & 0.83 & (1.76) & 0.83 & (1.83) & 0.79 & $(2.26)$ & 1.07 & 0.924 & 0.507 \\
\hline
\end{tabular}

SD: Standard deviation; IQR: Interquartile range; NTpro-BNP: N-terminal pro-B-type natriuretic peptide; ProADM: pro-adrenomedullin; hGH: human growth hormone; IGF-1: insulin-like growth factor 1; TSH: thyroid-stimulating hormone; $\mathrm{OR}^{*}=\log$ to the base 10 . 
In patients with syncope there is only few data about biomarkers. A recently published single-centre study showed, that NT-proBNP was a strong and independent diagnostic and prognostic marker in 161 patients hospitalized for syncope [17]. Troponin could also be identified as a prognostic predictor in older patients with syncope [18]. A recent published study showed, that $77 \%$ of patients presenting with syncope had detectable plasma troponin levels. Peak troponin concentration was associated with increasing risk of serious outcome and death [19]. We included all consecutive patients with syncopeall possible different causes, whether they were hospitalised or not. There was a wide range for age between 18 to 94 years, so we analysed data of the general population. In contrast to the literature we observed a less optimal performance of troponin in our cohort. Most of the patients with cardiovascular syncope $(82 \%)$ had a undetectable troponin level and we could not identifiy troponin as a good prognostic factor because there were no significant difference in the troponin levels compared between patients with and without serious adverse event.

In our study prealbumin and ProADM were the best markers to independently predict adverse events within 30 days in syncope patients. And ProADM levels were significantly higher for orthostatic and cardiovascular syncope than for neurally-mediated syncope, so ProADM may also play a role as a diagnostic marker (at least in the differentiation of harmless and dangerous causes of syncope). These results may be explained in that ProADM as a multifunctional peptide hormone reflects cardiovascular stress.

The role of prealbumin is not clear. Prealbumin (transthyretin) is a hepatic secretory protein, which reflects recent dietary intake and is also negative acute-phase reactant during inflammatory or other processes. Chertow showed in hemodialysis patients, that lower prealbumin levels were independently associated with mortality and hospitalisation [20].

Most likely it is a biomarker that reflects poor general state of health and risk of poor outcome at all.

\subsection{Limitations}

This is an observational study with all problems of review of medical records. Excluding patients with obvious severe or acute cardiac diseases could have led to some selection bias. The main limitation of our study is the relatively small sample size and the small number of adverse events. Even though the majority of events were not life-threatening illnesses such as acute myocardial infarction or death, we consider events such as rehospitalization relevant for the individual and from a public health perspective. In addition this is a single center study, therefore the generalization is restricted.

Because we measured biomarkers from residual blood, there were not always sufficient blood volume to measure for all biomarkers. Values for ProADM, TSH and hGH were not available in one case, cortisol and IGF-1 in two cases, vitamin B12, ferritin and transferrin in three cases, vitamin D in five cases, folic acid in ten cases and prealbumin in 18 cases.

\subsection{Conclusion}

This is the first report of prealbumin and ProADM as a prognostic factors in patients with syncope. In our study we found these biomarkers to correlate with adverse events and thus may improve risk assessment in patients with syncope. Randomized trials must clarify whether improved risk assessment with combined scores including the SFSR and biomarkers will reduce inappropriate hospitalizations and testing in syncope patients without affecting adverse events.

\section{REFERENCES}

[1] B. C. Sun, J. A. Emond and C. A. Camargo Jr., "Characteristics and Admission Patterns of Patients Presenting with Syncope to US Emergency Departments, 1992-2000," Academic Emergency Medicine, Vol. 11, No. 10, 2004, pp. 1029-1034.

[2] E. S. Soteriades, et al., "Incidence and Prognosis of Syncope," New England Journal of Medicine, Vol. 347, No. 12, 2002, pp. 878-885. doi:10.1056/NEJMoa012407

[3] A. Moya, et al., "Guidelines for the Diagnosis and Management of Syncope (Version 2009)," European Heart Journal, Vol. 30, No. 21, 2009, pp. 2631-2671.

[4] A. Bartoletti, et al., "Hospital Admission of Patients Referred to the Emergency Department for Syncope: A Single-Hospital Prospective Study Based on the Application of the European Society of Cardiology Guidelines on Syncope," European Heart Journal, Vol. 27, No. 1, 2006, pp. 83-88. doi:10.1093/eurheartj/ehi474

[5] M. L. Mendu, et al., "Yield of Diagnostic Tests in Evaluating Syncopal Episodes in Older Patients," Archives of Internal Medicine, Vol. 169, No. 14, 2009, pp. 1299-1305. doi:10.1001/archinternmed.2009.204

[6] B. C. Sun, J. A. Emond and C. A. Camargo Jr., "Direct Medical Costs of Syncope-Related Hospitalizations in the United States," American Journal of Cardiology, Vol. 95, No. 5, 2005, pp. 668-671. doi:10.1016/j.amjcard.2004.11.013

[7] T. P. Martin, B. H. Hanusa and W. N. Kapoor, "Risk Stratification of Patients with Syncope," Annals of Emergency Medicine, Vol. 29, No. 4, 1997, pp. 459-466. doi:10.1016/S0196-0644(97)70217-8

[8] F. Colivicchi, et al., "Development and Prospective Validation of a Risk Stratification System for Patients with Syncope in the Emergency Department: The OESIL Risk Score," European Heart Journal, Vol. 24, No. 9, 2003, pp. 811-819. doi:10.1016/S0195-668X(02)00827-8

[9] G. Costantino, et al., "Short- and Long-Term Prognosis 
of Syncope, Risk Factors, and Role of Hospital Admission: Results from the STePS (Short-Term Prognosis of Syncope) Study," Journal of the American College of Cardiology, Vol. 51, No. 3, 2008, pp. 276-283. doi:10.1016/j.jacc.2007.08.059

[10] J. Quinn, et al., "Prospective Validation of the San Francisco Syncope Rule to Predict Patients with Serious Outcomes," Annals of Emergency Medicine, Vol. 47, No. 5, 2006, pp. 448-454.

doi:10.1016/j.annemergmed.2005.11.019

[11] R. T. Saccilotto, et al., "San Francisco Syncope Rule to Predict Short-Term Serious Outcomes: A Systematic Review," Canadian Medical Association Journal, Vol. 183, No. 15, 2011, pp. E1116-E1126. doi:10.1503/cmaj.101326

[12] B. C. Sun, et al., "External Validation of the San Francisco Syncope Rule," Annals of Emergency Medicine, Vol. 49, No. 4, 2007, pp. 420-427. e1-e4.

[13] A. Birnbaum, et al., "Failure to Validate the San Francisco Syncope Rule in an Independent Emergency Department Population," Annals of Emergency Medicine, Vol. 52, No. 2, 2008, pp. 151-159. doi:10.1016/j.annemergmed.2007.12.007

[14] S. A. Grossman, et al., "Reducing Admissions Utilizing the Boston Syncope Criteria," Journal of Emergency Medicine, Vol. 42, No. 3, 2012, pp. 345-352. doi:10.1016/j.jemermed.2011.01.021
[15] A. Maisel, et al., "Midregion Prohormone Adrenomedullin and Prognosis in Patients Presenting with Acute Dyspnea: Results from the BACH (Biomarkers in Acute Heart Failure) Trial," Journal of the American College of Cardiology, Vol. 58, No. 10, 2011, pp. 1057-1067.

[16] O. S. Dhillon, et al., "Prognostic Value of Mid-Regional Pro-Adrenomedullin Levels Taken on Admission and Discharge in Non-ST-Elevation Myocardial Infarction: The LAMP (Leicester Acute Myocardial Infarction Peptide) II Study," Journal of the American College of Cardiology, Vol. 56, No. 2, 2010, pp. 125-133. doi:10.1016/i.jacc.2010.01.060

[17] R. Pfister, et al., "NT-Pro-BNP for Diagnostic and Prognostic Evaluation in Patients Hospitalized for Syncope," International Journal of Cardiology, Vol. 155, No. 2, 2012, pp. 268-272. doi:10.1016/j.ijcard.2010.10.013

[18] B. C. Sun, et al., "Predictors of 30-Day Serious Events in Older Patients with Syncope," Annals of Emergency Medicine, Vol. 54, No. 6, 2009, pp. 769-778. e1-e5.

[19] M. J. Reed, N. L. Mills and C. J. Weir, "Sensitive Troponin Assay Predicts Outcome in Syncope," Emergency Medicine Journal, Vol. 29, No. 12, 2012, pp. 1001-1003. doi:10.1136/emermed-2012-201332

[20] G. M. Chertow, et al., "Prealbumin, Mortality, and CauseSpecific Hospitalization in Hemodialysis Patients," Kidney International, Vol. 68, No. 6, 2005, pp. 2794-2800. doi:10.1111/j.1523-1755.2005.00751.x 